\title{
AVERAGE DENSITIES AND LINEAR RECTIFIABILITY OF MEASURES
}

\section{P. MÖRTERS}

\begin{abstract}
We show that a measure on $\mathbf{R}^{d}$ is linearly rectifiable if and only if the lower 1-density is positive and finite and agrees with the lower average 1-density almost everywhere.
\end{abstract}

\section{Introduction}

Let $\mu$ be a nonnegative, nonzero Radon measure on $\mathbb{R}^{d}$ and $\alpha \geq 0$. The lower $\alpha$-density of $\mu$ at $x$ is the number

$$
\underline{d}^{\alpha}(\mu, x)=\liminf _{t \downarrow 0} \frac{\mu(U(x, t))}{t^{\alpha}},
$$

where $U(x, t)$ denotes the open Euclidean ball centred in $x$ of radius $t$, and the upper $\alpha$-density of $\mu$ at $x$ is the number

$$
\bar{d}^{\alpha}(\mu, x)=\limsup _{t \downarrow 0} \frac{\mu(U(x, t))}{t^{\alpha}} .
$$

The geometric regularity of the measure $\mu$ is intimately related to the behaviour of the densities. We say $\mu$ is $\alpha$-rectifiable if $\mu$ is absolutely continuous with respect to $\alpha$-Hausdorff measure restricted to a countable family of smooth $\alpha$-manifolds and in the case of $\alpha=1$ we say that $\mu$ is linearly rectifiable. By Marstrand's Theorem (see [Mar64] or [Mat95, Chapter 14]) the equality

$$
0<\underline{d}^{\alpha}(\mu, x)=\bar{d}^{\alpha}(\mu, x)<\infty \mu \text {-almost everywhere }
$$

implies that $\alpha$ must be an integer and by Preiss' Regularity Theorem (see [Pre87] or [Mat95, Chapter 17]) such a measure $\mu$ is even $\alpha$-rectifiable.

A different type of density was introduced by Bedford and Fisher in [BF92], the so called average density or order-two density. Bedford and Fisher applied a logarithmic average to the density functions and defined the lower and upper average $\alpha$-density of $\mu$ at $x$ as

$$
\underline{D}^{\alpha}(\mu, x)=\liminf _{\varepsilon \rightarrow 0}(|\log \varepsilon|)^{-1} \int_{\varepsilon}^{1} \frac{\mu(U(x, t))}{t^{\alpha}} \frac{d t}{t}
$$

and

$$
\bar{D}^{\alpha}(\mu, x)=\limsup _{\varepsilon \rightarrow 0}(|\log \varepsilon|)^{-1} \int_{\varepsilon}^{1} \frac{\mu(U(x, t))}{t^{\alpha}} \frac{d t}{t} .
$$

We clearly have the following inequalities:

$$
\underline{d}^{\alpha}(\mu, x) \leq \underline{D}^{\alpha}(\mu, x) \leq \bar{D}^{\alpha}(\mu, x) \leq \bar{d}^{\alpha}(\mu, x) .
$$

It is natural to ask whether one can get statements about the geometric regularity of $\mu$ from weaker inequalities than (1), involving the average densities. This program was started by Falconer and Springer in [FS95] and their results were recently improved by Marstrand (see [Mar96]), who proved the following theorem: 
Theorem 1.1 Suppose $\mu$ is a nonnegative, nonzero Radon measure on $\mathbb{R}^{d}$ and $\alpha \geq 0$ such that

(i) $0<\bar{D}^{\alpha}(\mu, x)=\bar{d}^{\alpha}(\mu, x)<\infty$ for $\mu$-almost every $x$, or

(ii) $0<\underline{d}^{\alpha}(\mu, x)=\underline{D}^{\alpha}(\mu, x)<\infty$ for $\mu$-almost every $x$,

then $\alpha$ must be an integer.

In fact, Marstrand's proof in the case of the second condition needs the additional assumption that $\bar{d}^{\alpha}(\mu, x)<\infty$ for $\mu$-almost every $x$. This assumption is removed in [MP96] and a refinement of the argument given there is contained in Section 2 of this paper.

Do the inequalities above also imply $\alpha$-rectifiability of $\mu$ ? As Falconer and Springer point out, the answer is clearly no for the first inequality, even in the case $\alpha=1$, due to an example of O'Neil (see [O'N95]). It is the aim of this paper to give the following partial answer to this question in the case of the second inequality.

Theorem 1.2 Suppose $\mu$ is a nonnegative Radon measure on $\mathbb{R}^{d}$. Then

$$
0<\underline{d}^{1}(\mu, x)=\underline{D}^{1}(\mu, x)<\infty \text { for } \mu \text {-almost every } x
$$

if and only if $\mu$ is linearly rectifiable.

Of course, if $\mu$ is linearly rectifiable it is well known that $0<\underline{d}^{1}(\mu, x)=\bar{d}^{1}(\mu, x)<\infty$ for almost every $x$ and therefore it only remains to prove that (2) implies linear rectifiability of $\mu$. The proof of this statement consists of two parts. In Section 2 we employ the theory of tangent measure distributions to construct, from (ii), at almost every point $x$ an $\alpha$-flat tangent measure $\nu$ such that $\nu(U(0,1))=\underline{d}^{\alpha}(\mu, x)$ and in Section 3 we finish the proof by showing that in the case $\alpha=1$ such a tangent measure can only exist if $\mu$ is linearly rectifiable.

\section{Existence of Flat Tangent Measures}

We start by introducing the notion of tangent measures.

\section{Definition}

Let $\mathcal{M}\left(\mathbb{R}^{d}\right)$ be the set of nonnegative Radon measures on $\mathbb{R}^{d}$. Equipped with the vague topology, which is generated by the mappings $\mu \mapsto \int \varphi d \mu, \varphi$ continuous with compact support, $\mathcal{M}\left(\mathbb{R}^{d}\right)$ is a Polish space, see [Mat95, Chapter 14].

Let $0 \leq \alpha \leq d$ and $\mu \in \mathcal{M}\left(\mathbb{R}^{d}\right)$. For $r>0$ define $\mu_{x, r} \in \mathcal{M}\left(\mathbb{R}^{d}\right)$ to be the enlargement of $\mu$ at $x$ of factor $1 / r$, i.e. the measure defined by $\mu_{x, r}(A)=\mu(x+r A)$. The set $\operatorname{Tan}_{\alpha}(\mu, x)$ of $\alpha$-dimensional tangent measures of $\mu$ at $x$ is defined as the set of all limit points of $\mu_{x, r} / r^{\alpha}$ in the vague topology as $r \downarrow 0$.

A (tangent) measure $\nu$ is called $\alpha$-uniform if, for some $c>0, \nu(U(u, r))=c r^{\alpha}$ for all $r>0$ and $u$ in the support of $\nu . \nu$ is called $\alpha$-flat if $\alpha$ is an integer and there is a linear space $V \subseteq \mathbb{R}^{d}$ of dimension $\alpha$ and some $c>0$ such that $\nu=\left.c \cdot \mathcal{H}^{\alpha}\right|_{V}$, a multiple of the restriction of $\alpha$-Hausdorff measure to the space $V$. 
Preiss introduced the notion of tangent measures in his seminal paper [Pre87], he showed that if $0<\underline{d}^{\alpha}(\mu, x)=\bar{d}^{\alpha}(\mu, x)<\infty$ for $\mu$-almost every $x$, then at $\mu$-almost all $x$ all tangent measures of $\mu$ at $x$ are $\alpha$-flat. This implies that $\mu$ is $\alpha$-rectifiable.

In order to make quantitative statements about the set of tangent measures we introduce a family of probability distributions on $\operatorname{Tan}_{\alpha}(\mu, x)$, the so-called tangent measure distributions.

\section{Definition}

Let $\mu \in \mathcal{M}\left(\mathbb{R}^{d}\right)$ and $0 \leq \alpha \leq d$. For $x \in \mathbb{R}^{d}$ and $\varepsilon>0$ we define probability distributions $P_{\varepsilon}^{x}$ on $\mathcal{M}\left(\mathbb{R}^{d}\right)$ by

$$
P_{\varepsilon}^{x}(M)=(|\log \varepsilon|)^{-1} \int_{\varepsilon}^{1} 1_{M}\left(\frac{\mu_{x, r}}{r^{\alpha}}\right) \frac{d r}{r} \quad \text { for Borel sets } M \subseteq \mathcal{M}\left(\mathbb{R}^{d}\right) .
$$

$\mathcal{P}^{\alpha}(\mu, x)$ is defined as the set of all limit points of $\left(P_{\varepsilon}^{x}\right)_{\varepsilon>0}$ as $\varepsilon \downarrow 0$ in the weak topology, i.e. the topology generated by the mappings $P \mapsto \int F d P, F$ continuous and bounded. The elements of $\mathcal{P}^{\alpha}(\mu, x)$ are probability distributions on the set $\operatorname{Tan}_{\alpha}(\mu, x)$, they are the $\alpha$-dimensional tangent measure distributions of $\mu$ at $x$.

Tangent measure distributions were introduced by Bandt ([Ban92]) and Graf ([Gra95]) originally as a tool for the investigation of self-similar sets. They have also turned out to be valuable for the study of more general measures (see [Mör96], [MP96] or the thesis [Mör95]), which is due to the invariance properties described in the following theorem. For every $\lambda>0$ we define the rescaling operator $S_{\lambda}^{\alpha}: \mathcal{M}\left(\mathbb{R}^{d}\right) \rightarrow \mathcal{M}\left(\mathbb{R}^{d}\right)$ by $S_{\lambda}^{\alpha} \nu(E)=\left(1 / \lambda^{\alpha}\right) \cdot \nu(\lambda E)$ and for every $u \in \mathbb{R}^{d}$ we define the shift operator $T^{u}: \mathcal{M}\left(\mathbb{R}^{d}\right) \rightarrow \mathcal{M}\left(\mathbb{R}^{d}\right)$ by $T^{u} \nu(E)=\nu(u+E)$.

Theorem 2.1 Let $0 \leq \alpha \leq d$ and $\mu \in \mathcal{M}\left(\mathbb{R}^{d}\right)$.

(i) At every $x \in \mathbb{R}^{d}$ every tangent measure distribution $P \in \mathcal{P}^{\alpha}(\mu, x)$ fulfills

$$
P=P \circ\left(S_{\lambda}^{\alpha}\right)^{-1} \text { for all } \lambda>0 \text {. }
$$

(ii) At $\mu$-almost every $x$ every tangent measure distribution $P \in \mathcal{P}^{\alpha}(\mu, x)$ fulfills

$$
\iint G(\nu, u) d \nu(u) d P(\nu)=\iint G\left(T^{u} \nu,-u\right) d \nu(u) d P(\nu)
$$

for all Borel functions $G: \mathcal{M}\left(\mathbb{R}^{d}\right) \times \mathbb{R}^{d} \rightarrow[0, \infty)$.

Whereas the scaling invariance property (3) is easy to check, the shift invariance property (4) is quite difficult. For a proof, an interpretation and a number of applications of the formula (4) see [MP96]. In this paper we shall make use of the properties of tangent measure distributions by means of the following lemma.

Lemma 2.2 Suppose that a probability measure $P$ on $\mathcal{M}\left(\mathbb{R}^{d}\right)$ fulfills the scaling invariance property (3) for some $0 \leq \alpha \leq d$ and the shift-invariance property (4) and suppose that, with some $c>0, P$-almost every $\nu$ satisfies $\nu(U(0,1))=c$. Then $\alpha$ is an integer and P-almost every measure $\nu$ is $\alpha$-uniform and, in particular, $\alpha$-rectifiable. 
Proof From (3) we infer that, for every $r>0$,

$$
\begin{aligned}
P\left(\left\{\mu: \mu(U(0, r))=c r^{\alpha}\right\}\right) & =P \circ\left(S_{r}^{\alpha}\right)^{-1}(\{\mu: \mu(U(0,1))=c\}) \\
& =P(\{\mu: \mu(U(0,1))=c\})=1 .
\end{aligned}
$$

Thus, using (4) in the last step,

$$
\begin{aligned}
1 & =P\left(\left\{\mu: \mu(U(0, r))=c r^{\alpha}\right\}\right)=\frac{1}{c s^{\alpha}} \iint_{U(0, s)} 1_{\left\{\mu: \mu(U(0, r))=c r^{\alpha}\right\}}(\nu) d \nu(u) d P(\nu) \\
& =\frac{1}{c s^{\alpha}} \iint_{U(0, s)} 1_{\left\{\mu: \mu(U(u, r))=c r^{\alpha}\right\}}(\nu) d \nu(u) d P(\nu) .
\end{aligned}
$$

As this holds for arbitrary $r$ and $s$ we infer that, for $P$-almost every $\nu$, we have that $\nu(U(u, r))=c r^{\alpha}$ for $\nu$-almost every $u$ and every rational $r>0$. By continuity, $P$-almost every $\nu$ satisfies that $\nu(U(u, r))=c r^{\alpha}$ for every $u$ in the support of $\nu$ and every $r>0$, i.e. $\nu$ is $\alpha$-uniform. We can now use Marstrand's Theorem to conclude that $\alpha$ is an integer and Preiss' Regularity Theorem to conclude that $\nu$ is $\alpha$-rectifiable, but of course much weaker statements (like in [Kir88]) would suffice.

Theorem 2.3 Let $\mu \in \mathcal{M}\left(\mathbb{R}^{d}\right)$ be a measure such that

$$
0<\underline{d}^{\alpha}(\mu, x)=\underline{D}^{\alpha}(\mu, x)<\infty \text { for } \mu \text {-almost every } x .
$$

Then $\alpha$ is an integer and, at $\mu$-almost every $x$, there is an $\alpha$-flat measure $\nu \in \operatorname{Tan}_{\alpha}(\mu, x)$ such that

$$
\nu(U(0,1))=\underline{d}^{\alpha}(\mu, x) .
$$

Proof To make use of the preceding lemma, we prove that at $\mu$-almost every $x$ there is $P \in$ $\mathcal{P}^{\alpha}(\mu, x)$ such that $P$-almost every $\nu$ satisfies $\nu(U(0,1))=\underline{d}^{\alpha}(\mu, x)$.

Fix $x \in \mathbb{R}^{d}$ such that the density condition is fulfilled and choose $\varepsilon_{n} \downarrow 0$ such that

$$
\lim _{n \rightarrow \infty}\left(\left|\log \varepsilon_{n}\right|\right)^{-1} \int_{\varepsilon_{n}}^{1} \frac{\mu(U(x, r))}{r^{\alpha}} \frac{d r}{r}=\underline{D}^{\alpha}(\mu, x) .
$$

As, for all $m \geq 1$ and $R>0$,

$$
\begin{aligned}
P_{\varepsilon_{n}}^{x}\left(\left\{\nu: \nu(U(0, m))>R m^{\alpha}\right\}\right) & \leq(1 / R)\left(\left|\log \varepsilon_{n}\right|\right)^{-1} \int_{\varepsilon_{n}}^{1} \frac{\mu(U(x, m r))}{(m r)^{\alpha}} \frac{d r}{r} \\
& \leq(1 / R)\left(\left|\log \varepsilon_{n}\right|\right)^{-1} \int_{\varepsilon_{n}}^{m} \frac{\mu(U(x, r))}{r^{\alpha}} \frac{d r}{r}
\end{aligned}
$$

we can find, for every $\varepsilon>0$, a sequence $R_{m} \uparrow \infty$ such that, for all $n$,

$$
P_{\varepsilon_{n}}^{x}\left(\left\{\nu: \nu(U(0, m)) \leq R_{m} \text { for all } m=1,2, \ldots\right\}\right)>1-\varepsilon .
$$

This set is compact in $\mathcal{M}\left(\mathbb{R}^{d}\right)$ and hence, by Prohorov's Theorem, we can choose a convergent subsequence of $\left(P_{\varepsilon_{n}}^{x}\right)$ and denote the limit $P$. As the mapping $\nu \mapsto \nu(U(0,1))$ is lower semicontinuous we have

$$
\int \nu(U(0,1)) d P(\nu) \leq \lim _{n \rightarrow \infty}\left(\left|\log \varepsilon_{n}\right|\right)^{-1} \int_{\varepsilon_{n}}^{1} \frac{\mu(U(x, r))}{r^{\alpha}} \frac{d r}{r}=\underline{D}^{\alpha}(\mu, x)=\underline{d}^{\alpha}(\mu, x) .
$$


But $\nu(U(0,1)) \geq \underline{d}^{\alpha}(\mu, x)$ for all $\nu \in \operatorname{Tan}_{\alpha}(\mu, x)$ and therefore we must have $\nu(U(0,1))=\underline{d}^{\alpha}(\mu, x)$ for $P$-almost every $\nu$.

By Lemma $2.2 \alpha$ is an integer and, for $\mu$-almost every $x$, the $P \in \mathcal{P}^{\alpha}(\mu, x)$ we have constructed has the property that $P$-almost every $\nu \in \operatorname{Tan}_{\alpha}(\mu, x)$ is $\alpha$-rectifiable and fulfills $\nu(U(y, r))=\underline{d}^{\alpha}(\mu, x) \cdot r^{\alpha}$ for all $r>0$ and $y$ in the support of $\nu$. Fix such a tangent measure $\nu_{0} \in \operatorname{Tan}_{\alpha}(\mu, x)$ and, using that $\nu_{0}$ is $\alpha$-rectifiable, fix $y_{0}$ in its support such that all tangent measures of $\nu_{0}$ at $y_{0}$ are $\alpha$-flat. Since, for $\mu$-almost every $x, \operatorname{Tan}_{\alpha}(\nu, y) \subseteq \operatorname{Tan}_{\alpha}(\mu, x)$ for all $\nu \in \operatorname{Tan}_{\alpha}(\mu, x)$ and $y$ in the support of $\nu$ (see e.g. [Mat95, Theorem 14.16]), any tangent measure of $\nu_{0}$ at $y_{0}$ fulfills the requirements of the theorem.

\section{The Proof of Theorem 1.2}

We now look at the case $\alpha=1$ and show the following theorem.

Theorem 3.1 If $\mu \in \mathcal{M}\left(\mathbb{R}^{d}\right)$ is a measure such that at $\mu$-almost every $x$ there is a 1-flat measure $\nu \in \operatorname{Tan}_{1}(\mu, x)$ with $\nu(U(0,1))=\underline{d}^{1}(\mu, x)$, then $\mu$ is linearly rectifiable.

Clearly, Theorems 2.3 and 3.1 together imply Theorem 1.2. The following lemma contains the main ingredient of the proof of Theorem 3.1.

Lemma 3.2 For any $0<\xi<1 / 2-\sqrt{1 / 5}$ and $0<p \leq 1$ there is an $0<\varepsilon_{0}=\varepsilon_{0}(\xi, p)<1$ such that, for all $0<\varepsilon<\varepsilon_{0}$, whenever $E$ is a compact subset of the interval $[a, b]$ such that the Lebesgue measure of $[a, b] \backslash E$ is at least $p \cdot(b-a)$ and every connected component of the set $[a, b] \backslash E$ has length at most $\varepsilon \cdot(b-a)$, and whenever $\nu$ is a measure on the line such that $\nu([a, b] \backslash E) \leq \varepsilon \cdot(b-a)$ and

$$
\nu((x-t, x+t)) \geq t \text { for all } x \in E, 0<t \leq 2 \varepsilon \cdot(b-a),
$$

then we have

$$
\nu([a, b]) \geq(b-a) \cdot\left[\frac{1}{2}+\xi p\right]>\frac{b-a}{2} .
$$

Proof of Lemma 3.2. If $\xi$ and $p$ are given, we pick a number $\varrho$ such that $1 / 2+\xi<\varrho<1-\sqrt{1 / 5}$ and numbers $\varepsilon_{0}, \varepsilon_{1}>0$ such that

$$
\left(\varrho-\frac{1}{2}\right) \cdot\left(1-\varepsilon_{1}\right) p-\frac{\varepsilon_{1}}{2}-5 \varepsilon_{0} \geq \xi p .
$$

Suppose that $E$ and $\nu$ are given as in the formulation of the lemma and $0<\varepsilon<\varepsilon_{0}$. We denote by $\mathcal{I}$ the family of connected components of $[a, b] \backslash E$. We can pick a finite subfamily $\tilde{\mathcal{I}} \subseteq \mathcal{I}$ such that

$$
\sum_{I \in \tilde{\mathcal{I}}}|I| \geq\left(1-\varepsilon_{1}\right) \sum_{I \in \mathcal{I}}|I| \geq\left(1-\varepsilon_{1}\right) p \cdot(b-a) .
$$

For every $I \in \tilde{\mathcal{I}}$ we denote by $\bar{I}$ the interval consisting of all $x \in[a, b]$ such that the distance of $x$ to $I$ is at most $|I|$. We pick any of the longest $I \in \tilde{\mathcal{I}}$ and remove all $J \in \tilde{\mathcal{I}}$ with $J \subseteq \bar{I}$ 
from the collection. We can go on with this procedure, always starting with one of the longest remaining intervals which has not been considered and, after a finite number of steps, we have a new collection of intervals, which we order from left to right

$$
I_{1}<I_{2}<\cdots<I_{N-1}<I_{N}
$$

We now show how the statement of the lemma follows from

$$
\nu\left(\bigcup_{i=1}^{N} \bar{I}_{i}\right) \geq \varrho \cdot \mathcal{L}\left(\bigcup_{i=1}^{N} \bar{I}_{i}\right)-5 \varepsilon \cdot(b-a)
$$

where $\mathcal{L}$ denotes Lebesgue measure. Using Vitali's Covering Theorem (see e.g. [Mat95, Theorem 2.2]) we can cover $\mathcal{L}$-almost all of the set $E \backslash \bigcup_{i=1}^{N} \bar{I}_{i}$ by countably many disjoint intervals $\left[x_{i}-r_{i}, x_{i}+r_{i}\right]$ centred in $x_{i} \in E$ with $r_{i}<\varepsilon(b-a)$, which are contained in $[a, b] \backslash \bigcup_{i=1}^{N} \bar{I}_{i}$. Hence

$$
\nu\left([a, b] \backslash \bigcup_{i=1}^{N} \bar{I}_{i}\right) \geq \sum_{i=1}^{\infty} \nu\left(\left[x_{i}-r_{i}, x_{i}+r_{i}\right]\right) \geq \sum_{i=1}^{\infty} r_{i} \geq \frac{1}{2} \cdot \mathcal{L}\left(E \backslash \bigcup_{i=1}^{N} \bar{I}_{i}\right) .
$$

We also have

$$
\mathcal{L}\left(([a, b] \backslash E) \backslash \bigcup_{i=1}^{N} \bar{I}_{i}\right) \leq \mathcal{L}\left(\bigcup_{I \in \mathcal{I}} I \backslash \bigcup_{I \in \tilde{\mathcal{I}}} I\right) \leq \varepsilon_{1} \cdot(b-a) .
$$

Altogether we get

$$
\begin{aligned}
\nu([a, b]) & =\nu\left([a, b] \backslash \bigcup_{i=1}^{N} \bar{I}_{i}\right)+\nu\left(\bigcup_{i=1}^{N} \bar{I}_{i}\right) \\
& \geq \frac{1}{2} \cdot \mathcal{L}\left(E \backslash \bigcup_{i=1}^{N} \bar{I}_{i}\right)+\varrho \cdot \mathcal{L}\left(\bigcup_{i=1}^{N} \bar{I}_{i}\right)-5 \varepsilon(b-a) \\
& \geq \frac{1}{2} \cdot \mathcal{L}\left([a, b] \backslash \bigcup_{i=1}^{N} \bar{I}_{i}\right)+\varrho \cdot \mathcal{L}\left(\bigcup_{i=1}^{N} \bar{I}_{i}\right)-\varepsilon_{1}(b-a) / 2-5 \varepsilon(b-a) \\
& =\frac{b-a}{2}+\left[\varrho-\frac{1}{2}\right] \cdot \mathcal{L}\left(\bigcup_{i=1}^{N} \bar{I}_{i}\right)-\varepsilon_{1}(b-a) / 2-5 \varepsilon(b-a) \\
& \geq(b-a)\left[\frac{1}{2}+\left(\varrho-\frac{1}{2}\right) \cdot\left(1-\varepsilon_{1}\right) p-\varepsilon_{1} / 2-5 \varepsilon_{0}\right] \\
& \geq(b-a)\left[\frac{1}{2}+\xi p\right],
\end{aligned}
$$

using $\mathcal{L}\left(\bigcup_{i=1}^{N} \bar{I}_{i}\right) \geq \sum_{I \in \tilde{\mathcal{I}}}|I| \geq\left(1-\varepsilon_{1}\right) p \cdot(b-a)$ in the penultimate step and (5) in the final step.

It remains to show (7). For this purpose fix some $2 \leq k \leq N$. Denote by $Z_{k}$ the set of points between the intervals $I_{k-1}$ and $I_{k}$, such that the distance to one of the intervals is less than its length. Let

$$
C_{k}=I_{k-1} \cup Z_{k} \cup I_{k}
$$


We show that there are $\gamma_{2}, \ldots, \gamma_{N}$ with $\sum_{i=2}^{N} \gamma_{i} \leq \varepsilon(b-a)$ such that

$$
\nu\left(C_{k}\right) \geq \varrho\left[\frac{\left|I_{k-1}\right|}{2}+\frac{\left|I_{k}\right|}{2}+\left|Z_{k}\right|\right]-\gamma_{k} .
$$

If the distance of $I_{k-1}$ and $I_{k}$ is larger than the sum of their lengths, then the open interval centred in the right endpoint of $I_{k-1}$ of diameter $2\left|I_{k-1}\right|$ and the open interval centred in the left endpoint of $I_{k}$ of diameter $2\left|I_{k}\right|$ are disjoint and thus, using that the endpoints of these intervals are in $E$,

$$
\nu\left(C_{k}\right) \geq\left|I_{k-1}\right|+\left|I_{k}\right|=\frac{2}{3}\left[\frac{\left|I_{k-1}\right|}{2}+\frac{\left|I_{k}\right|}{2}+\left|Z_{k}\right|\right]>\varrho\left[\frac{\left|I_{k-1}\right|}{2}+\frac{\left|I_{k}\right|}{2}+\left|Z_{k}\right|\right] .
$$

We may thus suppose that $Z_{k}$ is an interval with $\left|Z_{k}\right| \leq\left|I_{k-1}\right|+\left|I_{k}\right|$ and that

$$
\nu\left(C_{k}\right)<\varrho\left[\frac{\left|I_{k-1}\right|}{2}+\frac{\left|I_{k}\right|}{2}+\left|Z_{k}\right|\right] .
$$

Looking at an open interval centred in the endpoint of the larger of the intervals $I_{k-1}$ and $I_{k}$ we get $\nu\left(C_{k}\right) \geq \frac{1}{2}\left(\left|I_{k-1}\right|+\left|I_{k}\right|\right)$. Together with (9) we conclude

$$
\left|Z_{k}\right|>\left(\frac{1-\varrho}{2 \varrho}\right)\left(\left|I_{k-1}\right|+\left|I_{k}\right|\right)
$$

If $\left|Z_{k}\right| \geq \varrho /(2-2 \varrho)\left(\left|I_{k-1}\right|+\left|I_{k}\right|\right)$, say $\left|Z_{k}\right|=\lambda\left(\left|I_{k-1}\right|+\left|I_{k}\right|\right)$ for some $1 \geq \lambda \geq \varrho /(2-2 \varrho)$, then

$$
\begin{aligned}
\nu\left(C_{k}\right) & \geq \lambda\left|I_{k-1}\right|+\lambda\left|I_{k}\right| \\
& =\varrho\left|Z_{k}\right|+(1-\varrho) \cdot \lambda\left(\left|I_{k-1}\right|+\left|I_{k}\right|\right) \\
& \geq \varrho\left[\frac{\left|I_{k-1}\right|}{2}+\frac{\left|I_{k}\right|}{2}+\left|Z_{k}\right|\right]
\end{aligned}
$$

contradicting (9). Hence

$$
\left|Z_{k}\right|<\left(\frac{\varrho}{2-2 \varrho}\right)\left(\left|I_{k-1}\right|+\left|I_{k}\right|\right)
$$

We know from the construction of the $I_{k}$ that $\zeta_{k}$, the centre of $C_{k}$, is in $Z_{k}$. Let us show that, with $\vartheta=1 / \varrho-3 / 2$, the interval $B=\left(\zeta_{k}-\vartheta\left|Z_{k}\right|, \zeta_{k}+\vartheta\left|Z_{k}\right|\right)$ contains no point of $E$.

For, if $y \in B \cap E$, let

$$
t=\frac{1}{2} \cdot\left(\left|I_{k-1}\right|+\left|I_{k}\right|\right)+\frac{1-2 \vartheta}{2}\left|Z_{k}\right|>0 .
$$

Then $(y-t, y+t) \subseteq C_{k}$ and, using (9),

$$
1 \leq \frac{\nu((y-t, y+t))}{t} \leq \frac{\nu\left(C_{k}\right)}{t}<\varrho \cdot \frac{\left|I_{k-1}\right|+\left|I_{k}\right|+2\left|Z_{k}\right|}{\left|I_{k-1}\right|+\left|I_{k}\right|+(1-2 \vartheta)\left|Z_{k}\right|}
$$

and hence, using (11),

$$
\left|Z_{k}\right|>\frac{1-\varrho}{2 \varrho-1+2 \vartheta}\left(\left|I_{k-1}\right|+\left|I_{k}\right|\right) \geq \frac{(1-\varrho)(2-2 \varrho)}{(2 \varrho-4+2 / \varrho) \varrho}\left|Z_{k}\right|=\left|Z_{k}\right|,
$$

a contradiction, which implies $B \cap E=\emptyset$. 
Observe that $B \subseteq Z_{k}$, since $\zeta_{k} \in B \cap Z_{k}$ but the boundaries of $I_{k-1}$ and $I_{k}$ are not. Hence there is a connected component $I \in \mathcal{I}$ of $[a, b] \backslash E$ such that

$$
B \subseteq I \subseteq Z_{k}
$$

Define

$$
r_{k}=\min \left\{\left|I_{k-1}\right|, 2 \vartheta\left|Z_{k}\right|\right\} \quad \text { and } \quad s_{k}=\min \left\{\left|I_{k}\right|, 2 \vartheta\left|Z_{k}\right|\right\} .
$$

As $|I| \geq 2 \vartheta\left|Z_{k}\right|$ the intersection of the open interval centred in the right endpoint of $I_{k-1}$ of radius $r_{k}$ and the open interval centred in the left endpoint of $I_{k}$ of radius $s_{k}$ is contained in $I$. Moreover, they are both contained in $C_{k}$ and therefore

$$
\nu\left(C_{k}\right)+\nu(I) \geq r_{k}+s_{k} .
$$

We choose $\gamma_{k}=\nu(I)$. We look at three cases that might occur in the definition of $s_{k}, r_{k}$.

(1) $r_{k}=\left|I_{k-1}\right|$ and $s_{k}=\left|I_{k}\right|$.

From (11) and (12) we derive

$$
\nu\left(C_{k}\right)+\gamma_{k} \geq\left|I_{k-1}\right|+\left|I_{k}\right| \geq \frac{\varrho}{2}\left(\left|I_{k-1}\right|+\left|I_{k}\right|\right)+(1-\varrho / 2) \frac{2-2 \varrho}{\varrho}\left|Z_{k}\right|,
$$

and $(8)$ follows since $(1-\varrho / 2)(2-2 \varrho) / \varrho \geq \varrho$.

(2) $r_{k}=\left|I_{k-1}\right|$ and $s_{k}=2 \vartheta\left|Z_{k}\right|$, or $r_{k}=2 \vartheta\left|Z_{k}\right|$ and $s_{k}=\left|I_{k}\right|$.

By symmetry we concentrate on the second case. Since $B \subseteq Z_{k}$ we have

$$
\left|I_{k-1}\right| \leq \frac{1}{2}\left(\left|I_{k-1}\right|+\left|I_{k}\right|+\left|Z_{k}\right|\right)-\vartheta\left|Z_{k}\right|
$$

and thus

$$
\left|I_{k}\right| \geq\left|I_{k-1}\right|-(1-2 \vartheta)\left|Z_{k}\right| \text {. }
$$

Using this, (12) and (11) we conclude

$$
\begin{aligned}
\nu\left(C_{k}\right)+\gamma_{k} & \geq 2 \vartheta\left|Z_{k}\right|+\frac{1}{2}\left|I_{k}\right|+\frac{1}{2}\left|I_{k-1}\right|-(1 / 2-\vartheta)\left|Z_{k}\right| \\
& \geq \frac{\varrho}{2}\left(\left|I_{k-1}\right|+\left|I_{k}\right|\right)+\left[(1 / 2-\varrho / 2) \frac{2-2 \varrho}{\varrho}+(3 \vartheta-1 / 2)\right]\left|Z_{k}\right|,
\end{aligned}
$$

and $(8)$ follows from $(1 / 2-\varrho / 2)(2-2 \varrho) / \varrho+(3 \vartheta-1 / 2) \geq \varrho$.

(3) $r_{k}=s_{k}=2 \vartheta\left|Z_{k}\right|$.

From (12) and (10) we derive

$$
\nu\left(C_{k}\right)+\gamma_{k} \geq 4 \vartheta\left|Z_{k}\right| \geq \varrho\left|Z_{k}\right|+\left(\frac{4}{\varrho}-6-\varrho\right) \frac{1-\varrho}{2 \varrho}\left(\left|I_{k-1}\right|+\left|I_{k}\right|\right),
$$

and (8) follows since $\varrho<1-\sqrt{1 / 5}$ implies $(4 / \varrho-6-\varrho)(1-\varrho) /(2 \varrho) \geq \varrho / 2$. 
In all three cases we have verified (8) for a $\gamma_{k}$ such that the $\gamma_{2}, \ldots, \gamma_{N}$ fulfill

$$
\sum_{i=2}^{N} \gamma_{i} \leq \sum_{I \in \mathcal{I}} \nu(I)=\nu([a, b] \backslash E) \leq \varepsilon(b-a) .
$$

To finish the proof we estimate, taking special care of the "boundary" intervals $I_{1}$ and $I_{N}$,

$$
\begin{aligned}
\nu\left(\bigcup_{i=1}^{N} \bar{I}_{i}\right) & \geq \sum_{k=2}^{N} \nu\left(C_{k}\right)-\sum_{k=1}^{N} \nu\left(I_{k}\right) \\
& \geq \varrho \cdot\left[\sum_{k=2}^{N} \frac{\left|I_{k-1}\right|}{2}+\frac{\left|I_{k}\right|}{2}+\left|Z_{k}\right|\right]-\sum_{k=2}^{N} \gamma_{k}-\varepsilon \cdot(b-a) \\
& \geq \varrho \cdot \mathcal{L}\left(\bigcup_{k=1}^{N} \bar{I}_{k}\right)-\frac{3}{2}\left(\left|I_{1}\right|+\left|I_{N}\right|\right)-2 \varepsilon \cdot(b-a) \\
& \geq \varrho \cdot \mathcal{L}\left(\bigcup_{k=1}^{N} \bar{I}_{k}\right)-5 \varepsilon \cdot(b-a),
\end{aligned}
$$

which is (7) and thus the proof is finished.

We now have the means to carry out the Proof of Theorem 3.1.

Denote by $B(x, r)$ the closed Euclidean ball of radius $r$ centred in $x$.

Suppose the statement is false. Then there is some $0<\eta<1$ and $\delta>0$ such that the set

$$
\left\{x \in \mathbb{R}^{d}: \delta<\underline{d}^{1}(\mu, x)<\eta \cdot \bar{d}^{1}(\mu, x)\right\}
$$

has positive measure. Fix some $0<\xi<1 / 2-\sqrt{1 / 5}$ and $1>1-p>\eta$. We can then pick $0<\varepsilon<\varepsilon_{0}(\xi, p)$, such that $\varepsilon<\delta$ and $\eta(1+5 \varepsilon)<1-p$ and

$$
\frac{1}{2}\left(1+\frac{\varepsilon}{\delta-\varepsilon}\right)(1+5 \varepsilon)<\frac{1}{2}+\xi p,
$$

and we can find a compact set $F \subseteq \mathbb{R}^{d}$ with $\mu(F)>0$ and numbers $0<d<D<\infty$ with $\delta<d<\eta D$ such that there is $R>0$ such that for all $x \in F$

- $\mu(U(x, r))>(d-\varepsilon) r$ for all $0<r<R$,

- there is a sequence $r_{n} \downarrow 0$ such that the tangent measure

$$
\tilde{\mu}=\lim _{n \rightarrow \infty} \frac{\mu_{x, r_{n}}}{r_{n}}
$$

is 1 -flat and $\mu\left(B\left(x, r_{n}\right)\right)<d \cdot r_{n}$, and

- there is a sequence $s_{n} \downarrow 0$ such that $\mu\left(U\left(x, s_{n}\right)\right)>D \cdot s_{n}$.

Using the Density Theorem (see e.g. [Mat95, Corollary 2.14]) we can fix a density point $y \in F$, i.e. a point $y \in F$ such that $\lim _{r \rightarrow 0} \mu(B(y, r) \backslash F) / \mu(B(y, r))=0$. Then, in particular,

$$
\lim _{n \rightarrow \infty} \frac{1}{r_{n}} \mu\left(B\left(y, r_{n}\right) \backslash F\right)=0 \text {. }
$$


Denote by $L_{y}$ the line through $y$ such that $\tilde{\mu}=\left.c \cdot \mathcal{H}^{1}\right|_{L_{y}-y}$ and by $\pi_{y}$ the orthogonal projection onto $L_{y}$. Denote by $S(y, r)$ the set of all points $x \in B(y, r)$ such that the distance of $x$ to its projection onto $L_{y}$ is at most $r \varepsilon /(1+5 \varepsilon)$ and, similarly, denote by $S(0,1)$ the set of all $x \in B(0,1)$ such that the distance of $x$ to its projection onto $L_{y}-y$ is at most $\varepsilon /(1+5 \varepsilon)$. Then

$$
\limsup _{n \rightarrow \infty} \frac{1}{r_{n}} \mu\left(B\left(y, r_{n}\right) \backslash S\left(y, r_{n}\right)\right)=\limsup _{n \rightarrow \infty} \frac{\mu_{y, r_{n}}}{r_{n}}(B(0,1) \backslash S(0,1))=0 .
$$

We may partition $\left(L_{y}-y\right) \cap B(0,1)$ into finitely many disjoint intervals $J_{1}, \ldots, J_{k}$ with $0<$ $\left|J_{i}\right|<\varepsilon /(1+5 \varepsilon)$ and for each interval we can choose a nonnegative continuous function $f_{i}$ on $\mathbb{R}^{d}$ which is positive in some point of $J_{i}$ with support contained in the set of those points in $S(0,1)$ whose projection onto $L_{y}-y$ hits $J_{i}$. We find

$$
0<\int f_{i}(z) d \tilde{\mu}(z)=\lim _{n \rightarrow \infty} \frac{1}{r_{n}} \int_{F} f_{i}\left(\frac{z-y}{r_{n}}\right) d \mu(z)
$$

and conclude that there is $N$ such that for all $n \geq N$ and every $1 \leq i \leq k$ the set $\pi_{y}^{-1}\left(y+r_{n} J_{i}\right) \cap$ $S\left(y, r_{n}\right)$ contains points of $F$. Putting these facts together we can find $0<r<R / 4$ such that

- every connected component of the set $\pi_{y}(B(y, r)) \backslash \pi_{y}(F \cap S(y, r))$ has length less than $r \cdot 2 \varepsilon /(1+5 \varepsilon)$,

- there is an open set $B \supseteq B(y, r)$ such that $\mu(B) \leq d \cdot r$,

- $\mu(B(y, r) \backslash S(y, r)) \leq \frac{(d-\varepsilon) \cdot \varepsilon}{1+5 \varepsilon} \cdot r$

- $\mu(B(y, r) \backslash F) \leq \frac{(d-\varepsilon) \cdot \varepsilon}{1+5 \varepsilon} \cdot r$.

Let $s=r /(1+5 \varepsilon)$. We define a compact subset of the line $L_{y}$ by

$$
E_{y}=\pi_{y}(F \cap S(y, r)) \cap B(y, s) \subseteq L_{y} .
$$

We now show that the hypotheses of Lemma 3.2 are fulfilled with $E_{y}$ in the rôle of $E$ and $\pi_{y}(B(y, s))$ in the rôle of $[a, b]$. By choice of $s$ the connected components of $\pi_{y}(B(y, s)) \backslash E_{y}$ have length less than $\varepsilon(2 s)$.

By Besicovitch's Covering Theorem (see e.g. [Mat95, Theorem 2.8]) we can cover $\mu$-almost all of $F \cap B(y, r)$ with a countable family $(B(x(i), s(i)))$ of disjoint balls centred in $F$ and contained in $B$ such that $\mu(B(x(i), s(i))) \geq D \cdot s(i)$. We infer that

$$
\sum_{i=1}^{\infty} s(i) \leq \frac{\mu(B)}{D} \leq \frac{d r}{D}<\eta r .
$$

Hence the set $E_{y}$ covers at most a proportion of $\eta(1+5 \varepsilon)<1-p$ of the length of $\pi_{y}(B(y, s))$. In other words,

$$
\mathcal{L}\left(\pi_{y}(B(y, s)) \backslash E_{y}\right) \geq 2 s(1-\eta(1+5 \varepsilon)) \geq p(2 s) .
$$

Now define a measure on $L_{y}$ by

$$
\nu=\left.\frac{1}{d-\varepsilon} \cdot \mu\right|_{B(y, r)} \circ \pi_{y}^{-1} .
$$


We have

$$
\nu\left(\pi_{y}(B(y, s)) \backslash E_{y}\right) \leq 1 /(d-\varepsilon) \cdot[\mu(B(y, r) \backslash F)+\mu(B(y, r) \backslash S(y, r))] \leq \varepsilon(2 s) .
$$

Finally, for all $x \in E_{y}$ and $t \leq 4 \varepsilon s<R$, there is $\tilde{x} \in F \cap S(y, r) \cap \pi_{y}^{-1}(x)$. As $U(\tilde{x}, t) \subseteq$ $U(x, 5 \varepsilon s) \subseteq B(y, r)$ we get

$$
\nu\left(U(x, t) \cap L_{y}\right) \geq \frac{1}{d-\varepsilon} \cdot \mu(U(\tilde{x}, t)) \geq t,
$$

and thus the hypotheses of Lemma 3.2 are fulfilled. Therefore

$$
\nu\left(\pi_{y}(B(y, s))\right) \geq(2 s) \cdot\left[\frac{1}{2}+\xi p\right] .
$$

On the other hand, from the construction of $\nu$, we get

$$
\begin{aligned}
\nu\left(\pi_{y}(B(y, s))\right) & \leq \frac{1}{d-\varepsilon} \cdot \mu(B(y, r)) \\
& \leq \frac{d}{d-\varepsilon}(1+5 \varepsilon) s .
\end{aligned}
$$

Now (14) and (15) together imply

$$
\frac{1}{2}\left(1+\frac{\varepsilon}{d-\varepsilon}\right)(1+5 \varepsilon) \geq\left[\frac{1}{2}+\xi p\right]
$$

which contradicts (13) and finishes the proof.

\section{Remarks:}

(i) There are alternative ways to prove Theorem 3.1 from Lemma 3.2. For example one could, instead of showing the existence of densities, use the fact that, if a set $F$ does not contain a linearly rectifiable subset of positive measure, the projections onto almost all lines have Lebesgue measure zero by the Besicovitch-Federer Projection Theorem (see [Mat95, Chapter 18]) and then apply Lemma 3.2 with $p=1$.

(ii) Our result naturally raises the question for which dimensions (other than 1) a result like Theorem 3.1 and hence Theorem 1.2 holds. This seems to be a delicate question.

\section{References}

[Ban92] C. BAndT, The tangent distribution for self-similar measures. Lecture at the 5th Conference on Real Analysis and Measure Theory, Capri, 1992.

[BF92] T. Bedford And A.M. Fisher, Analogues of the Lebesgue density theorem for fractal sets of reals and integers. Proc. London Math. Soc.(3), 64:95-124, 1992.

[FS95] K.J. Falconer and O.B. Springer, Order-two density of sets and measures with non-integral dimension, Mathematika, 42:1-14, 1995. 
[Gra95] S. Graf, On Bandt's tangential distribution for self-similar measures. Mh. Math., $120: 223-246,1995$.

[Kir88] B. Kirchneim, Uniformly distributed measures, tangent measures and analytic varieties. In: Proc. Conf. Topology and Measure V, Wissenschaftliche Beiträge der ErnstMoritz-Arndt-Universität Greifswald, pp. 54-60, 1988.

[Mar64] J.M. Marstrand, The $(\phi, s)$-regular subsets of $n$-space. Trans. Amer. Math. Soc., 113:369-392, 1964.

[Mar96] J.M. Marstrand, Order-two density and the strong law of large numbers, Mathematika, 43:1-22, 1996.

[Mat95] P. Mattila, The Geometry of Sets and Measures in Euclidean Spaces. Cambridge University Press, Cambridge, 1995.

[Mör95] P. Mörters, Tangent Measure Distributions and the Geometry of Measures. PhD thesis, University College London, 1995.

[Mör96] P. MörTERs, Symmetry properties of average densities and tangent measure distributions of measures on the line. To appear in Adv. Math., 1996.

[MP96] P. Mörters And D. Preiss, Tangent measure distributions of fractal measures. Preprint, 1996.

[O'N95] T.C. O'NeIL, A Local Version of the Projection Theorem and Other Results in Geometric Measure Theory. PhD thesis, University College London, 1995.

[Pre87] D. Preiss, Geometry of measures in $\mathbb{R}^{n}$ : Distribution, rectifiability and densities. Ann. Math., 125:537-643, 1987.

Dr. P. Mörters

Universität Kaiserslautern

Fachbereich Mathematik

67663 Kaiserslautern

Germany.

\section{A75: MEASURE AND INTEGRATION:}

Classical measure theory; Length, area, volume, other geometric measure theory. 